\title{
Radiotherapy versus combination radiotherapy-bevacizumab for the treatment of recurrent high-grade glioma: a systematic review
}

\author{
Daniel P. Kulinich ${ }^{1,2}$. John P. Sheppard ${ }^{1,2} \cdot$ Thien Nguyen $^{1,2} \cdot$ Aditya M. Kondajji ${ }^{1,2}$ • Ansley Unterberger ${ }^{1,2}$. \\ Courtney Duong ${ }^{1,2} \cdot$ Adam Enomoto $^{1,2} \cdot$ Kunal Patel $^{1,2} \cdot$ Isaac Yang ${ }^{1,2,3,4,5,6,7}$ (D)
}

Received: 2 November 2020 / Accepted: 25 February 2021 / Published online: 2 April 2021

(C) The Author(s) 2021

\begin{abstract}
Background High-grade gliomas (HGG) comprise the most common primary adult brain cancers and universally recur. Combination of re-irradiation therapy (reRT) and bevacizumab (BVZ) therapy for recurrent HGG is common, but its reported efficacy is mixed.

Objective To assess clinical outcomes after reRT \pm BVZ in recurrent HGG patients receiving stereotactic radiosurgery (SRS), hypofractionated radiosurgery (HFSRT), or fully fractionated radiotherapy (FFRT).

Methods We performed a systematic review of PubMed, Web of Science, Scopus, Embase, and Cochrane databases, following the Preferred Reporting Items for Systematic Reviews and Meta-Analyses (PRISMA) guidelines. We identified studies reporting outcomes for patients with recurrent HGG treated via reRT \pm BVZ. Cohorts were stratified by BVZ treatment status and reirradiation modality (SRS, HFSRT, and FFRT). Outcome variables were overall survival (OS), progression-free survival (PFS), and radiation necrosis ( $\mathrm{RN})$.

Results Data on 1399 patients was analyzed, with 954 patients receiving reRT alone and 445 patients receiving reRT + BVZ. All patients initially underwent standard-of-care therapy for their primary HGG. In a multivariate analysis that adjusted for median patient age, WHO grade, RT dosing, reRT fractionation regimen, time between primary and re-irradiation, and re-irradiation target volume, BVZ therapy was associated with significantly improved OS $(2.51,95 \% \mathrm{CI}[0.11,4.92]$ months, $P=.041)$ but no significant improvement in PFS $(1.40,95 \%$ CI $[-0.36,3.18]$ months, $P=.099)$. Patients receiving BVZ also had significantly lower rates of RN $(2.2 \%$ vs $6.5 \%, P<.001)$.

Conclusions Combination of reRT + BVZ may improve OS and reduce RN rates in recurrent HGG, but further controlled studies are needed to confirm these effects.
\end{abstract}

Keywords Radiotherapy $\cdot$ Radiosurgery $\cdot$ Recurrent $\cdot$ Glioma $\cdot$ Astrocytoma $\cdot$ Glioblastoma $\cdot$ Bevacizumab

This article is part of the Topical Collection on Brain Tumors

Isaac Yang

iyang@mednet.ucla.edu

1 Departments of Neurosurgery, University of California, Los Angeles, 300 Stein Plaza, Suite 562, Los Angeles, CA, USA

2 David Geffen School of Medicine, Los Angeles (UCLA), Los Angeles, CA, USA

3 Departments of Radiation Oncology, University of California, Los Angeles, Los Angeles, CA, USA

4 Departments of Head and Neck Surgery, University of California, Los Angeles, Los Angeles, CA, USA

5 Jonsson Comprehensive Cancer Center, Los Angeles, CA, USA

6 Los Angeles Biomedical Research Institute, Los Angeles, CA, USA

7 Harbor-UCLA Medical Center, West Carson, CA, USA

\section{Introduction}

Glioblastoma (GBM) and anaplastic astrocytoma comprise the majority of gliomas. While incurable, a growing armamentarium of treatment options requires complex interdisciplinary decision-making to determine optimal management strategies based on individual patient-care goals. Classically, the World Health Organization (WHO) has histologically classified high-grade gliomas (HGGs) as WHO grade III gliomas, which include anaplastic astrocytomas (AA) and oligodendrogliomas (AOA), and WHO grade IV glioblastoma [41]. GBM accounts for approximately $60 \%$ of $\mathrm{HGG}$ and $50 \%$ of all malignant brain tumors and portends the poorest prognosis [19]. Since then, WHO glioma classification has been re-examined and re-defined according to various genetic 
markers, mainly IDH mutation and $1 \mathrm{p} / 19 \mathrm{q}$ deletion status [41]. The current standard-of-care for GBM involves a multimodal approach, including surgical resection of the primary lesion, chemotherapy with temozolomide, and adjuvant radiotherapy (typically involving conventional external beam radiation therapy, EBRT). Landmark studies by Stupp and colleagues demonstrated improved 2-year survival to $27.2 \%$ from $10.9 \%$ when adding EBRT to temozolomide (TMZ) chemotherapy in primary GBM [71, 72]. Even with modern treatment regimens, however, HGG recurrence is virtually inevitable. Estimated post-recurrence survival in AA and GBM patients is approximately 10 and 6 months, respectively [72]. Thus, there is a need for further clinical investigation of survival outcomes to optimize treatment protocols for recurrent HGG.

Eighty percent of recurrent HGG tumors appear within $2 \mathrm{~cm}$ of the initial contrast-enhanced primary lesion [48]. Recent advances in imaging and radiotherapy techniques allow irradiation with higher doses, improved local tumor control, and sparing of adjacent tissue [1]. Several fractionation regimens are available, which offer flexibility between the number of treatment sessions and maximum radiation doses required, including stereotactic radiosurgery (SRS), hypofractionated stereotactic radiotherapy (HFSRT), and fully fractionated radiotherapy (FFRT) [64, 66]. HFSRT and FFRT regimens effectively target larger-volume lesions with lower doses administered over cumulative fractions, while SRS may offer a favorable radiation modality for smaller lesions [56]. Recurrent HGG is regularly treated with a combination of repeat surgical resection, systemic cancer therapies, and radiosurgery. Reresection and repeat radiation therapy (reRT) improve overall survival (OS) and progression-free survival (PFS) in recurrent HGG; however, lesion size and proximity to eloquent tissue may limit reRT use [64]. Adjuvant chemotherapy and radiosensitizing agents may further improve outcomes in the setting of reRT, but their effect on survival remains inconclusive [17, 62]. Furthermore, the reported efficacy of traditional chemotherapy agents such as TMZ is limited to patients with nonresistant, MGMT-methylated primary, or recurrent tumors [53, 65]. Overall, despite a plethora of new therapeutic alternatives, there remains no established standardized treatment protocol for recurrent $\operatorname{HGG}[35,64]$.

One promising avenue for glioma treatment involves immunotherapy targeting tumor blood supply. HGG survives, differentiates, and grows well in hypoxic niches, which upregulates a conglomerate of molecular factors in the glial tumor cells, including hypoxic inhibitory factor (HIF)-1 and 2 [2]. HIF is a potent inducer of vascular endothelial growth factor (VEGF), a transcription factor key to promoting vasculogenesis. Neurovasculature in hypoxic tumor niches is tortuous and aberrant; it jeopardizes the blood-brain barrier and promotes further damage, edema, and necrosis. AntiVEGF therapy with bevacizumab (BVZ) can reduce aberrant vasculogenesis, reduce radiation necrosis, and improve outcomes in clinical studies of HGG [5, 27, 28, 40]. Beyond its use in primary lesions, BVZ is combined with reRT (reRT + $\mathrm{BVZ}$ ) to treat recurrent $\mathrm{HGG}$. Recent studies have found that reRT + BVZ regimens are well tolerated, reduce radiation necrosis (RN) [23], and improve survival outcomes in HGG patients $[8,18,29]$. However, due to mixed results [14, 23], there remains no consensus on the utility of reRT + BVZ regimens for HGG. Here, we present a systematic review that synthesizes data from the published literature to assess the efficacy of reRT + BVZ treatment for recurrent HGG compared to reRT alone.

\section{Methods}

Adherence to the Preferred Reporting Items for Systematic Reviews and Meta-Analyses (PRISMA) (www.prismastatement.org) was maintained throughout this study. We indexed peer-reviewed abstracts and articles published between 1990 and 2019 in the following databases: PubMed, Scopus, Embase, Cochrane, and Web of Science. The last electronic search was completed in November 2019. An intersectional Boolean-search was performed to screen for articles with search terms including "high-grade glioma," "glioblastoma," "recurrent," or "irradiation" AND (or NOT) "bevacizumab." For inclusion in our systematic review, we required that articles met the following eligibility criteria: full-text, English-language clinical trials, prospective, or retrospective studies of patients with histologically proven, recurrent HGG who had initially received standard surgery and chemoradiation therapy for their primary lesion, with eventual tumor recurrence treated with either reRT or reRT + BVZ. Articles describing patients who received additional systemic therapies with reRT other than standard chemotherapy or BVZ, or who received reRT modalities other than SRS, HFSRT, or FFRT (e.g., brachytherapy), were excluded.

We further queried the bibliographies of identified manuscripts to screen for additional articles appropriate for review that could have escaped our electronic search. Some publications reported multiple treatment cohorts (e.g., both reRT and reRT + BVZ); in these cases, we separately extracted corresponding data for each treatment regimen. Where applicable, we required that authors controlled for demographic variables between groups in studies reporting aggregate demographic data across multiple treatment groups. Studies without reported demographic comparisons were excluded. Some articles reported overall survival data for WHO grades III/IV individually without reporting cumulative outcome data; in these cases, we only included data for GBM patients.

The following measures were extracted from included studies: (1) patient demographics (age, sex, and Karnofsky Performance Scale (KPS) at start of reRT), (2) 
WHO grade (III/IV), (3) primary RT and other adjuvant therapy parameters, (4) reRT and details of other therapies administered after recurrence, (5) latency from initial RT to reRT, (6) planned tumor volume for reRT (PTV). Extracted outcome measures included (1) overall survival after reRT (OS), (2) progression-free survival after reRT (PFS), (3) rates of radiation necrosis $(\mathrm{RN})$, and (4) treatment-induced toxicity rates. For studies with multiple reRT protocols that qualified under a specific modality (SRS if $<5$ fractions administered, HFSRT if 5-10 fractions administered, or FFRT if $>10$ fractions administered), radiation dosage was computed as the average of the individual regimens. For comparative purposes, we converted radiation doses to reflect an equivalent total dose in 2 Gy fractions (EQD2) utilizing a linear-quadratic model with $\alpha / \beta=2$ [24]. Of note, IDH mutation and $1 \mathrm{p} / 19 \mathrm{q}$ deletion status were not extracted since most publications did not classify according to the updated WHO glioma classification [41].

\section{Statistical analysis}

We utilized unpaired Welch $t$-tests and Fischer exact tests for pairwise comparisons of continuous and binary variables. BVZ treatment and reRT fractionation modality were the two primary explanatory variables-of-interest. To compare OS and PFS between BVZ treatment groups (reRT vs. reRT + BVZ) and between reRT radiation modalities (SRS vs. HFSRT vs. FFRT), we used weighted Welch $t$-tests in order to weigh individual studies appropriately by their relative sample size when estimating pooled differences. Finally, we employed multivariate linear regression analysis to evaluate the amount of variance in OS and PFS explained collectively by all explanatory variables considered (median age at reRT, GBM diagnosis, BVZ treatment status, reRT fractionation modality, time between initial RT and reRT, planned target volume (PTV) for reRT, and EQD2 at both initial- and reirradiation). All statistical analysis was performed in R, using the $\mathrm{R}$ core utilities in addition to the weights package for $\mathrm{R}$ $[54,59]$. Statistical significance is defined as $P<.05$.

To further assess the quality of reviewed studies and the validity of our systematic review, we used standard metaanalytic methods to quantify cross-study heterogeneity and assess the risk of study bias (RoB) on OS and RN outcomes. We separately fit generalized linear mixed models within each treatment group that incorporated random study effects. These models allowed for the calculation of $H, \tau^{2}$, and $I^{2}$, standard, and closely related metrics for quantifying heterogeneity in a meta-analysis. Statistical significance of study heterogeneity was determined for each treatment group based upon Cochran's $Q$ statistic. To assess the potential influence of RoB, we determined a numeric RoB score for each reviewed study which incorporated key factors that could increase each study's susceptibility to bias. Estimation of RoB scores is fully described in Supplementary Table 3 and considered the overall rigor of described study methodology, whether studies were prospective, randomized, or blinded, as well as the variability in patients' tumor subtypes, chemotherapy, reRT, immunotherapy regimens, stringency of RN diagnosis, and adequacy of follow-up. Regression analyses were performed for each treatment group to detect any associations between RoB score and reported outcomes. We used simple linear regression (i.e., Pearson's correlations) to assess the impact of RoB on OS or PFS within each treatment group, given the limited number of studies reporting standard errors or confidence intervals for medial survival estimates. For RN outcomes, we used meta-regression to assess the impact of RoB score as this more robust approach also accounted for random study effects. Meta-analyses were performed using the meta, metafor, and metamedian packages for $\mathrm{R}[45,63,75]$. The metamedian package was used to quantify heterogeneity in reported OS outcomes for studies which at minimum reported the range (min and max) of survival times in addition to medial survival, using quantile estimation methods described by McGrath et al. $[44,45]$. We did not attempt to quantify heterogeneity in reported PFS outcomes, given the paucity of studies amenable to quantile estimation.

\section{Results}

Our combined electronic and manual bibliographic search identified 1742 articles in total before eligibility screening. Of these, 293 articles survived to the full-text review stage, of which 34 papers (2\%) were deemed eligible for inclusion in our review (Fig. 1). Among the included studies, 26 papers reported data on patients receiving reRT alone, and 12 papers reported data on patients receiving reRT + BVZ (Tables 1 and 2) $[3,6-8,10,13-16,18,20,22,23,26,29-31,33,34,36,37$, $42,46,51,57,61,67-69,74,76,77,79,80]$.

\section{Demographic variables}

\section{reRT group}

We identified 954 patients receiving reRT alone. Ninety-four patients $(10 \%)$ received FFRT, $206(21 \%)$ received HFSRT, and $654(69 \%)$ received SRS. Among 862 patients for whom gender was reported, 492 were male and 370 were female $(M$ $=57 \%, F=43 \%)$. Specifically, FFRT had 39 female patients (43\%), HFSRT had 74 female patients (42\%), and SRS had 257 female patients (43\%). Among patients receiving reRT, $779(82 \%)$ patients had GBM, and 175 (18\%) had WHO grade III gliomas. reRT patients had a median Karnofsky Performance Score (KPS) of 80 (range: 40-100) (Table 3). 
Fig. 1 PRISMA flow diagram

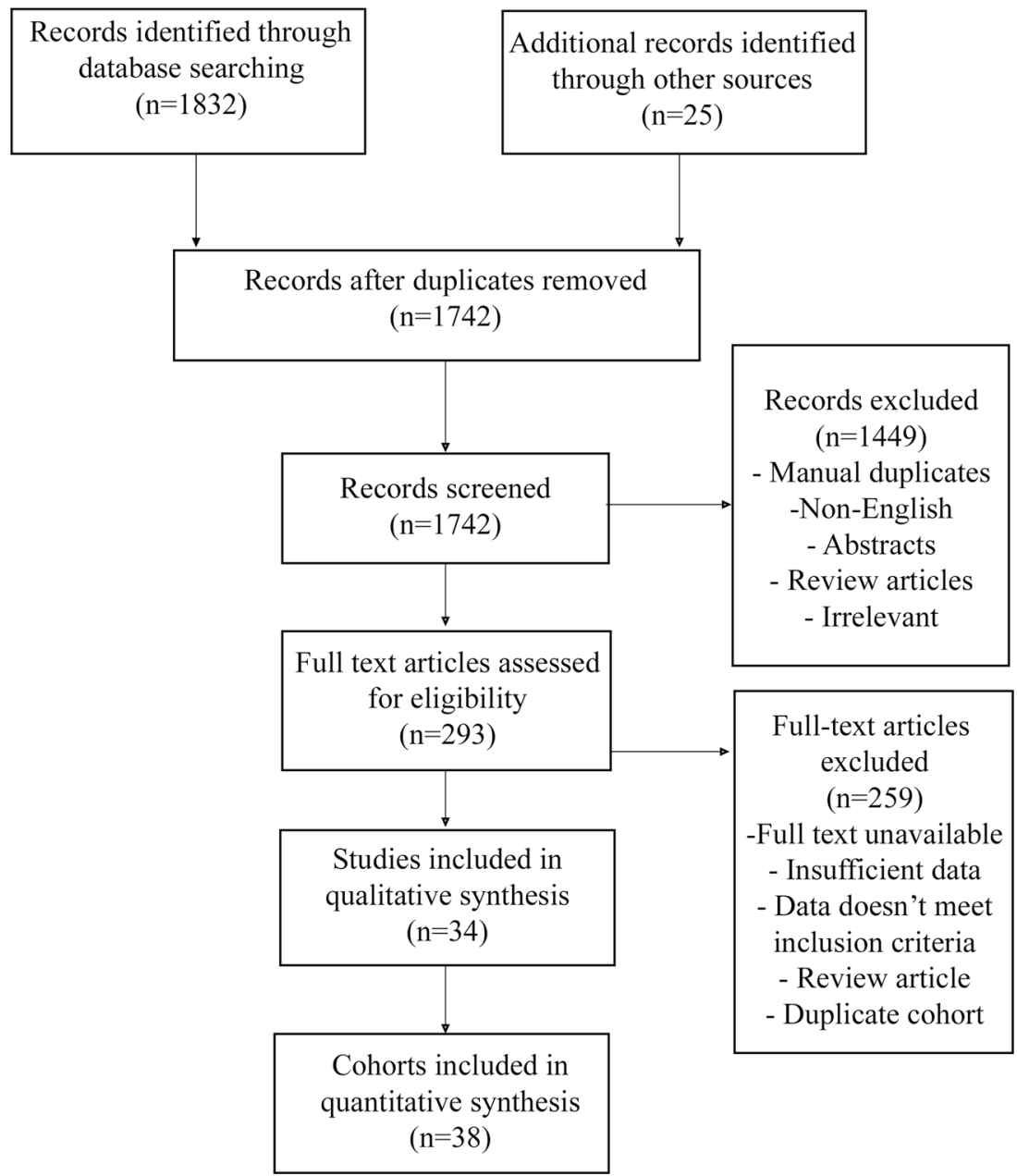

reRT + BVZ group

Four hundred forty-five patients receiving reRT + BVZ were identified; 293 (66\%) received FFRT, 80 (18\%) received HFSRT, and 72 (16\%) received SRS. Among 453 patients for whom gender was reported, 299 were male and 154 were female $(M=66 \%, F=34 \%)$. Specifically, FFRT had 106 female patients $(35 \%, P=.17)$, HFSRT had 24 female patients (36\%, $P=.38)$, and SRS had 17 female patients $(24 \%, P=$ .002). Among patients receiving reRT + BVZ, 337 (76\%) had GBM, and 108 (24\%) had WHO grade III gliomas, with a median KPS of 80 (range: 40-100) (Table 3).

\section{Treatment parameters}

All patients had either WHO grade III or IV tumors and had previously received primary surgical resection followed by RT with a median cumulative dose of 60 Gy and a median fractional dose of 2 Gy. All patients received chemotherapy for their primary lesion, with the most received agent being TMZ (> 54\%). Patients receiving reRT alone underwent salvage reRT after a mean latency of $13.4 \pm 5.3$ months (range:
3-40 months), compared to $16.2 \pm 5.9$ months (range: 8-40 months, $P=.18$ ) for patients receiving reRT $+\mathrm{BVZ}$ (Table 4). reRT + BVZ patients received adjuvant BVZ dosed at 10 $\mathrm{mg} / \mathrm{kg}$ once every two weeks for a range of $2-12$ cycles.

Within the reRT group, patients receiving SRS incurred lower cumulative doses at reRT $(19.7 \pm 6.2 \mathrm{~Gy})$ than patients receiving HFSRT $(28.2 \pm 3.8 \mathrm{~Gy}, P=.0053)$ or FFRT $(37.9 \pm$ $3.2 \mathrm{~Gy} ; P=.0058)$. There were no differences in total dose at re-irradiation among patients receiving different modalities within the reRT + BVZ treatment group (Table 4). PTV was comparable between reRT and reRT + BVZ groups after stratifying by reRT modality. PTV for reRT vs. reRT + BVZ patients averaged $120.5 \mathrm{~cm}^{3}$ vs. $102.9 \mathrm{~cm}^{3}$ for FFRT $(P=$ $.91), 26.6 \mathrm{~cm}^{3}$ vs. $33.0 \mathrm{~cm}^{3}$ for HFSRT $(P=.83)$, and 11.9 $\mathrm{cm}^{3}$ vs. $3.8 \mathrm{~cm}^{3}$ for SRS $(P=.14)$, respectively (Table 4). However, within the reRT + BVZ group, PTV was significantly different between each radiation modality group (Table 4). The latency between initial radiotherapy and reirradiation only differed between reRT and reRT + BVZ patients who received SRS, which averaged 20.5 months for reRT + BVZ patients compared to 12.2 months for reRT patients $(P<.001)$. Other demographic variables and radiation 
Table 1 Studies on re-irradiation with bevacizumab for recurrent high-grade glioma

\begin{tabular}{|c|c|c|c|c|c|c|c|c|c|c|c|c|c|}
\hline \multirow[t]{2}{*}{ Study } & \multirow[t]{2}{*}{ Modality } & \multirow{2}{*}{$\begin{array}{l}\text { Patients } \\
\text { (grade III/ } \\
\text { IV) }\end{array}$} & \multirow{2}{*}{$\begin{array}{l}\text { Median } \\
\text { age (y) }\end{array}$} & \multirow{2}{*}{$\begin{array}{l}\text { Initial } \\
\text { radiation* } \\
(\mathrm{Gy})\end{array}$} & \multirow{2}{*}{$\begin{array}{l}\text { FU } \\
(\mathrm{mo})\end{array}$} & \multicolumn{2}{|c|}{ Re-irradiation } & \multirow{2}{*}{$\begin{array}{l}\text { Median } \\
\text { KPS }\end{array}$} & \multirow{2}{*}{$\begin{array}{l}\text { Median } \\
\text { PTV } \\
\left(\mathrm{cm}^{3}\right)\end{array}$} & \multirow{2}{*}{$\begin{array}{l}\text { Median } \\
\text { OS (mo) }\end{array}$} & \multirow{2}{*}{$\begin{array}{l}\text { Median } \\
\text { PFS (mo) }\end{array}$} & \multirow{2}{*}{$\begin{array}{l}\mathrm{RN} \\
(N)\end{array}$} & \multirow{2}{*}{$\begin{array}{l}\text { Total } \\
\text { toxicity } \\
(N)\end{array}$} \\
\hline & & & & & & $*(\mathrm{~Gy})$ & $\begin{array}{l}\text { EQD2 } \\
\text { (Gy) }\end{array}$ & & & & & & \\
\hline $\begin{array}{r}\text { Fleischmann } \\
2019[23]\end{array}$ & FFRT & $124(29 / 95)$ & 51 & $60 / 2$ & 17 & $36 / 2$ & 36 & 80 & 117 & 9.0 & 5.0 & 6 & 12 \\
\hline $\begin{array}{l}\text { Palmer }^{\gamma} \\
2018[51]\end{array}$ & FFRT & $68(14 / 48)$ & 57 & $60 / 2$ & 11 & $35 / 3.5$ & 48 & 80 & 35 & 13.9 & - & 0 & 7 \\
\hline $\begin{array}{l}\text { Palmer }^{\gamma} \\
2018 \text { [51] }\end{array}$ & FFRT & $50(5 / 39)$ & 54 & $60 / 2$ & 8 & $35 / 3.5$ & 48 & 80 & 35 & 13.3 & - & 0 & 7 \\
\hline $\begin{array}{l}\text { Schernberg } \\
2017[61]\end{array}$ & FFRT & $35(11 / 24)$ & 57 & $60 / 2$ & 22 & $56 / 3.2$ & 83 & - & 104 & 10.5 & 6.7 & 0 & 0 \\
\hline $\begin{array}{c}\text { Back } 2015 \\
{[3]}\end{array}$ & FFRT & $18(5 / 13)$ & 50 & $60 / 2$ & 15 & $35 / 2.0$ & 30 & - & 136 & 10.0 & - & 1 & 2 \\
\hline $\begin{array}{r}\text { Hundsberger } \\
2013 \text { [34] }\end{array}$ & FFRT & $10(4 / 6)$ & 45 & $60 / 2$ & 41 & $42 / 2.6$ & 58 & 70 & 190 & 8.4 & 5.7 & 0 & 4 \\
\hline $\begin{array}{l}\text { Yasuda } 2018 \\
\text { [79] }\end{array}$ & HFSRT & $29(7 / 22)$ & 46 & $60 / 2$ & 19 & $42 / 6$ & 84 & 80 & 34 & 10.4 & 5.6 & 0 & 3 \\
\hline $\begin{array}{l}\text { Minniti } 2015 \\
\text { [46] }\end{array}$ & HFSRT & $26(7 / 19)$ & 50 & $60 / 2$ & 13 & $25 / 5$ & 44 & - & 31 & 11.0 & - & 0 & 4 \\
\hline $\begin{array}{l}\text { Gutin } 2009 \\
\text { [29] }\end{array}$ & HFSRT & $25(5 / 20)$ & 56 & $59 / 2$ & 15 & $30 / 6$ & 60 & 80 & 34 & 12.5 & 7.3 & 0 & 31 \\
\hline $\begin{array}{l}\text { Clarke } 2017 \\
\text { [14] }\end{array}$ & SRS & $15(5 / 10)$ & 63 & $60 / 2$ & - & $33 / 11$ & 107 & 90 & - & 13.0 & 7.0 & 1 & 14 \\
\hline $\begin{array}{c}\text { Cabrera } 2013 \\
\text { [8] }\end{array}$ & SRS & $15(7 / 8)$ & 53 & $60 / 2$ & 20 & $15 / 15$ & 56 & 90 & 3 & 14.4 & 3.9 & 0 & 15 \\
\hline $\begin{array}{l}\text { Cuneo } 2012 \\
{[18]}\end{array}$ & SRS & $42(9 / 33)$ & 47 & $60 / 2$ & 21 & $17 / 9$ & 48 & 80 & 5 & 11.2 & 5.2 & 2 & 17 \\
\hline
\end{tabular}

$F F R T$, fully fractionated radiotherapy; $H F S R T$, hypofractionated stereotactic radiotherapy; SRS, stereotactic radiosurgery; $F U$, median latency between initial and re-irradiation; $E Q D 2$, equivalent dose in $2 \mathrm{~Gy}$ per fraction; $K P S$, Karnofsky performance scale; $P T V$, planned tumor volume; $O S$, overall survival from re-irradiation; $P F S$, progression-free survival from re-irradiation; $R N$, radiation necrosis; $y$, year; mo, months; $N$, number

*Total dose/fraction dose

${ }^{\gamma}$ Same publication with separate cohorts

parameters did not differ significantly by treatment group or reRT modality (Tables 3 and 4).

\section{Clinical outcomes}

The reRT treatment group had a mean OS of $9.9 \pm 2.1$ months, with PFS of $5.2 \pm 1.6$ months and $\mathrm{RN}$ rate of $9.5 \%$ (95\% CI $[7.7 \%, 11.6 \%])$. In comparison, the reRT + BVZ group had a mean OS of $11.2 \pm 2.1$ months $(P=.057)$, with PFS of $5.6 \pm$ 1.0 months $(P=.55)$, and an average $\mathrm{RN}$ rate of $2.2 \%(95 \% \mathrm{CI}$ $[1.1 \%, 4.0 \%], P<.001)$ (Table 5).

Among patients receiving FFRT, the reRT treatment group had a mean OS of $8.7 \pm 1.6$ months, with PFS of $5.3 \pm 0.5$ months, and $\mathrm{RN}$ rate of $6.4 \%$ (95\% CI [2.4\%, 13.4\%]). In comparison, the reRT + BVZ group had a mean OS of 11.0 \pm 2.4 months $(P=.095)$, with PFS of $5.4 \pm 0.8$ months $(P=$ .38 ), and RN rate of $2.3 \%$ (95\% CI [0.9\%, $4.7 \%], P=.088$ ) (Table 5).

Among patients receiving HFSRT, the reRT group had a mean OS of $9.4 \pm 1.6$ months, with PFS of $5.2 \pm 1.4$ months, and $\mathrm{RN}$ rate of $7.7 \%$ (95\% CI [4.5\%, 12.2\%]). In comparison, the reRT + BVZ treatment group had a mean OS of $11.3 \pm 1.6$ months $(P=.045)$, with PFS of $6.4 \pm 0.9$ months $(P=.40)$, and $\mathrm{RN}$ rate of $0 \%(95 \% \mathrm{CI}[0 \%, 4.5 \%], P=.0076)$ (Table 5).

Among patients receiving SRS, the reRT treatment group had a mean OS of $10.3 \pm 2.2$ months, with PFS of $5.2 \pm 2.1$ months, and RN of $6.2 \%$ (95\% CI [2.4\%, 10.0\%]). The reRT $+\mathrm{BVZ}$ group had a mean OS of $12.2 \pm 1.8$ months $(P=.11)$, mean PFS of $5.3 \pm 0.9$ months $(P=.92)$, and $\mathrm{RN}$ rate of $4.2 \%$ (95\% CI [0.9\%, 11.7\%], $P=.097$ ) (Table 5).

Tables 6 and 7 present the results of multivariate regression analysis assessing the significance of nine explanatory variables-of-interest accounting for the variance in OS and PFS, respectively. BVZ treatment status was the only significant predictor of OS $(P=.041)$ (Table 6). No significant predictor variables were identified for PFS (Table 7).

\section{Heterogeneity analysis}

Analysis of study heterogeneity was performed for RN and OS (Supplementary Table 1,2). Fixed and random effect model values are reported side by side. For both outcomes, 
Table 2 Studies on re-irradiation alone for recurrent high-grade glioma

\begin{tabular}{|c|c|c|c|c|c|c|c|c|c|c|c|c|c|}
\hline \multirow[t]{2}{*}{ Study } & \multirow[t]{2}{*}{ Modality } & \multirow{2}{*}{$\begin{array}{l}\text { Patients } \\
\text { (grade III/ } \\
\text { IV) }\end{array}$} & \multirow{2}{*}{$\begin{array}{l}\text { Median } \\
\text { age (y) }\end{array}$} & \multirow{2}{*}{$\begin{array}{l}\text { Initial } \\
\text { radiation* } \\
\text { (Gy) }\end{array}$} & \multirow{2}{*}{$\begin{array}{l}\mathrm{FU} \\
(\mathrm{mo})\end{array}$} & \multicolumn{2}{|c|}{ Re-irradiation } & \multirow{2}{*}{$\begin{array}{l}\text { Median } \\
\text { KPS }\end{array}$} & \multirow{2}{*}{$\begin{array}{l}\text { Median } \\
\text { PTV } \\
\left(\mathrm{cm}^{3}\right)\end{array}$} & \multirow{2}{*}{$\begin{array}{l}\text { Median } \\
\text { OS (mo) }\end{array}$} & \multirow{2}{*}{$\begin{array}{l}\text { Median } \\
\text { PFS } \\
\text { (mo) }\end{array}$} & \multirow{2}{*}{$\begin{array}{l}\mathrm{RN} \\
(N)\end{array}$} & \multirow{2}{*}{$\begin{array}{l}\text { Total } \\
\text { toxicity } \\
(N)\end{array}$} \\
\hline & & & & & & $*(\mathrm{~Gy})$ & $\begin{array}{l}\text { EQD2 } \\
\text { (Gy) }\end{array}$ & & & & & & \\
\hline $\begin{array}{r}\text { Fleischmann } \\
2019[23]\end{array}$ & FFRT & $37(8 / 29)$ & 51 & $60 / 2$ & 18 & $36 / 2$ & 36 & 80 & 122 & 9.0 & 5.0 & 5 & 14 \\
\hline $\begin{array}{c}\text { Hundsberger } \\
2013 \text { [34] }\end{array}$ & FFRT & $4(2 / 2)$ & 45 & $60 / 2$ & 41 & $42 / 2.6$ & 58 & 70 & 190 & 14.3 & 3.7 & 1 & - \\
\hline Combs 2005 [17] & FFRT & $53(0 / 53)$ & 55 & $57 / 2$ & 10 & $36 / 2$ & 36 & - & 49 & 8.0 & 5.0 & 0 & - \\
\hline $\begin{array}{l}\text { Gigliotti } 2018 \\
\text { [26] }\end{array}$ & HFSRT & $25(5 / 25)$ & 54 & $60 / 2$ & 18 & $25 / 5$ & 44 & - & 10 & 9.0 & - & 0 & - \\
\hline Zemlin 2018 [80] & HFSRT & $41(6 / 35)$ & 56 & $60 / 2$ & 22 & $31 / 4$ & 30 & - & 63 & 6.7 & 4.3 & 2 & - \\
\hline Holt 2016 [31] & HFSRT & $34(0 / 34)$ & 60 & $60 / 2$ & 14 & $23 / 3$ & 30 & - & 9 & 10.9 & 7.1 & 1 & 5 \\
\hline $\begin{array}{l}\text { Dincoglan } 2015 \\
\quad[20]\end{array}$ & HFSRT & $28(0 / 28)$ & 56 & $60 / 2$ & 11 & $25 / 5$ & 44 & 80 & 37 & 10.3 & 5.8 & 3 & 8 \\
\hline $\begin{array}{l}\text { Ciammella } 2013 \\
\text { [13] }\end{array}$ & HFSRT & $15(0 / 15)$ & 52 & $60 / 2$ & 11 & $25 / 5$ & 44 & 90 & - & 9.5 & - & 0 & - \\
\hline $\begin{array}{l}\text { Vordermark } 2005 \\
\text { [76] }\end{array}$ & HFSRT & $14(0 / 14)$ & 50 & $60 / 2$ & 19 & $30 / 5$ & 53 & 90 & 15 & 7.9 & 4.9 & 0 & 19 \\
\hline $\begin{array}{l}\text { Voynov } 2002 \\
\text { [77] }\end{array}$ & HFSRT & $10(4 / 4)$ & 48 & $60 / 2$ & 18 & $30 / 5$ & 53 & 80 & - & 10.1 & - & 6 & - \\
\hline Hudes 1999 [33] & HFSRT & $20(1 / 19)$ & 52 & $60 / 2$ & 3 & $30 / 3$ & 38 & 80 & - & 10.5 & - & 0 & 2 \\
\hline $\begin{array}{l}\text { Shepherd } 1997 \\
\text { [67] }\end{array}$ & HFSRT & $21(11 / 10)$ & 37 & $55 / 2$ & 29 & $35 / 3$ & 53 & - & - & 10.7 & - & 4 & 0 \\
\hline Bir 2015 [6] & SRS & $36(0 / 36)$ & 53 & $60 / 2$ & 7 & $28 / 28$ & 210 & - & - & 7.3 & - & 2 & 14 \\
\hline Pinzi 2015 [57] & SRS & $\begin{array}{l}128 \\
(40 / 88)\end{array}$ & 51 & $60 / 2$ & 15 & $20 / 10$ & 69 & - & 5 & 11.5 & - & 7 & 19 \\
\hline $\begin{array}{l}\text { Martinez-Carrillo } \\
2014 \text { [42] }\end{array}$ & SRS & $87(41 / 46)$ & 49 & $60 / 2$ & 14 & $18 / 18$ & 90 & 83 & - & 10.0 & - & 0 & - \\
\hline Khalil 2013 [36] & SRS & $50(16 / 34)$ & 58 & $60 / 2$ & 10 & $15 / 15$ & 64 & 70 & - & 11.4 & 8.6 & 7 & 10 \\
\hline Cuneo 2012 [18] & SRS & $21(5 / 16)$ & 48 & $60 / 2$ & 19 & $17 / 9$ & 47 & 80 & 6 & 3.9 & 2.1 & 4 & 11 \\
\hline Skeie 2012 [69] & SRS & $32(0 / 32)$ & 51 & $60 / 2$ & 20 & $31 / 31$ & 256 & 73 & - & 12.0 & - & 0 & 2 \\
\hline Elliott 2011 [22] & SRS & $26(10 / 16)$ & 60 & $60 / 2$ & 8 & $30 / 30$ & 240 & 90 & - & 13.5 & - & 2 & 3 \\
\hline Torok 2011 [74] & SRS & $14(0 / 14)$ & 58 & $60 / 2$ & 13 & $24 / 12$ & 84 & - & - & 10.0 & 5.0 & 0 & 0 \\
\hline Biswas 2009 [7] & SRS & $33(0 / 33)$ & 58 & $60 / 2$ & 9 & $15 / 15$ & 64 & - & - & 6.7 & 4.3 & 1 & - \\
\hline Kong 2008 [37] & SRS & $65(0 / 65)$ & 49 & $60 / 2$ & - & $16 / 16$ & 72 & 70 & 11 & 13.0 & 4.6 & 22 & - \\
\hline Combs 2005 [15] & SRS & $32(0 / 32)$ & 56 & $54 / 2$ & 10 & $15 / 15$ & 64 & - & 10 & 10.0 & 5.0 & 0 & 0 \\
\hline Hall 1995 [30] & SRS & $35(8 / 26)$ & 48 & $60 / 2$ & 8 & $20 / 20$ & 110 & 70 & 28 & 8.0 & - & 5 & 0 \\
\hline Shrieve 1995 [68] & SRS & $76(4 / 72)$ & 46 & $60 / 2$ & 10 & $13 / 13$ & 49 & 80 & - & 10.2 & - & 0 & - \\
\hline $\begin{array}{c}\text { Chamberlain } \\
1994[10]\end{array}$ & SRS & $20(14 / 6)$ & 34 & $60 / 2$ & 11 & $14 / 14$ & 52 & 80 & - & 8.0 & 4.0 & 0 & 7 \\
\hline
\end{tabular}

$F F R T$, fully fractionated radiotherapy; HFSRT, hypofractionated stereotactic radiotherapy; $S R S$, stereotactic radiosurgery; $F U$, median latency between initial and re-irradiation; $E Q D 2$, equivalent dose in $2 \mathrm{~Gy}$ per fraction; $K P S$, Karnofsky performance scale; $P T V$, planned tumor volume; $O S$, overall survival from re-irradiation; $P F S$, progression-free survival from re-irradiation; $R N$, radiation necrosis; $y$, year; $m o$, months; $N$, number

*Total dose/fraction dose

studies were separated according to re-treatment protocols. For RN outcomes, heterogeneity was significant among FFRT $(Q(2)=10.82 ; P=.0045)$, HFSRT $(Q(8)=34.8 ; P<$ $.0001)$ and SRS treatment groups $(Q(12)=54.66 ; P<.0001)$. For OS outcomes, heterogeneity was significant among FFRT $+\operatorname{BVZ}(Q(2)=6.27 ; P=0.044)$, HFSRT $(Q(3)=9.85 ; P=$ $.02)$, and SRS $(Q(8)=45.73 ; P<.0001)$ groups.

\section{Risk of bias analysis}

We assessed individual studies for risk of bias (RoB) using ten factors of study quality for reRT + BVZ studies (Supplementary Table 3), and nine parameters of study quality for reRT-only studies (Supplementary Table 4; see Methods for further details). The average RoB score for reRT $+\mathrm{BVZ}$ 
Table 3 Treatment group demographics

\begin{tabular}{|c|c|c|c|c|c|c|c|c|c|c|c|}
\hline \multirow[t]{2}{*}{ Modality } & \multicolumn{2}{|l|}{ Patients } & \multicolumn{3}{|l|}{ Age } & \multicolumn{3}{|c|}{ WHO glioma grade } & \multicolumn{3}{|c|}{ Karnofsky Performance Score } \\
\hline & $N(\mathrm{M} / \mathrm{F})$ & $P$ value* & Mean & SE & $P$ value* & III & IV & $P$ value* $[95 \% \mathrm{CI}]$ & Median & Range & $P$ value* \\
\hline Cumulative $^{\mathrm{i}}$ & $954(492 / 370)$ & - & 51.3 & 6.3 & - & $175(18.3 \%)$ & $779(81.7 \%)$ & - & 80 & $40-100$ & - \\
\hline FFRT $^{\mathrm{i}}$ & $94(50 / 39)$ & - & 50.3 & 5.0 & - & $10(10.6 \%)$ & $84(89.4 \%)$ & - & 75 & $60-100$ & - \\
\hline HFSRT $^{\mathrm{i}}$ & $206(99 / 74)$ & - & 51.6 & 6.5 & - & $27(13.1 \%)$ & $179(86.9 \%)$ & - & 80 & $60-100$ & - \\
\hline $\mathrm{SRS}^{\mathrm{i}}$ & $654(343 / 257)$ & - & 51.3 & 6.8 & - & $138(21.1 \%)$ & $516(78.9 \%)$ & - & 80 & $40-100$ & - \\
\hline Cumulative $\mathrm{i}^{\mathrm{ii}}$ & $445(299 / 154)$ & .002 & 52.4 & 5.3 & .60 & $108(24.3 \%)$ & $337(75.7 \%)$ & $.012[0.53,0.93]$ & 80 & $40-100$ & .48 \\
\hline FFRT $^{\mathrm{ii}}$ & $293(195 / 106)$ & .17 & 52.3 & 4.7 & .57 & $68(23.2 \%)$ & $225(76.8 \%)$ & $.0076[0.17,0.82]$ & 80 & $40-100$ & .22 \\
\hline HFSRT $^{\text {ii }}$ & $80(42 / 24)$ & .38 & 50.7 & 5.0 & .83 & $19(23.8 \%)$ & $61(76.3 \%)$ & $.032[0.24,0.993]$ & 80 & $70-100$ & .21 \\
\hline SRS $^{\mathrm{ii}}$ & $72(55 / 17)$ & .002 & 54.3 & 8.1 & .51 & $21(29.2 \%)$ & $51(70.8 \%)$ & $.13[0.37,1.18]$ & 90 & $50-100$ & .042 \\
\hline
\end{tabular}

$M$, male; $F$, female; $S E$, standard error; $W H O$, World Health Organization; FFRT, fully fractionated radiotherapy; HFSRT, hypofractionated stereotactic radiotherapy, $S R S$; stereotactic radiosurgery; $C I$, confidence interval; "Cumulative" denotes combined statistic for all fractionation groups within a treatment group; $m o$, months; $N$, number

*Pairwise comparison between treatment groups

${ }^{\mathrm{i}}$ Re-irradiation only group

${ }^{i i}$ Re-irradiation with bevacizumab group

studies was $7.75 \pm 1.83$ (range: $4-10$ ), and $7.31 \pm 1.61$ (range: 4-10) for reRT-only studies. We evaluated the effects of RoB scores on outcome measures using Pearson correlations for PFS and RN and meta-regression for RN (Supplementary Table 5). The only significant association of outcomes with RoB score was observed for reported PFS among the FFRT group $(r(1)=-1.0 ; P<.0001)$.

\section{Discussion}

High-grade gliomas remain incurable with universal recurrence. Treatment of recurrent HGG is limited and heterogeneous in terms of treatment protocols and reported efficacy. Although salvage combination therapy with re-irradiation and adjuvant bevacizumab (BVZ) has mixed outcomes, multiple studies suggest it improved overall survival, progression-free survival, radiation necrosis, and tolerable toxicity in patients with recurrent HGG. The current study attempts to consolidate knowledge from the available published data on this topic.

The reported outcome data compares recurrent HGG patients receiving reRT with or without concomitant BVZ. We set strict inclusion criteria that eliminate any cohorts wherein more than $17.5 \%$ of patients received additional systemic therapies or adjuvant surgery at the time of reRT. We found that patients receiving BVZ had marginally improved average overall survival $(P=.057)$ and significantly lower rates of radiation necrosis $(P<0.001)$ compared to patients receiving reRT alone. Stratifying by the reRT fractionation regimen, BVZ significantly improves OS $(P=.045)$ and $\mathrm{RN}(P=$ .0076) for HFSRT patients. Despite similar trends towards improved OS and RN in reRT + BVZ patients undergoing
FFRT and SRS, no effects reached statistical significance. In a multivariate analysis adjusting for median patient age, WHO grade (III vs. IV), reRT modality, latency between initial RT and reRT, planned tumor volume for radiation planning, EQD2 at reRT, and total EQD2 across initial RT and reRT, treatment with BVZ was the only significant predictor of improved overall survival and accounted for improved OS of 2.5 months on average (95\% CI [0.1, 4.9], $P=.041)$.

The foremost accepted therapeutic mechanism for BVZ is the mitigation of radiation necrosis secondary to irradiationinduced vascular dysfunction [81]. First, irradiation of glioma tissue causes vascular damage and subsequent hypoxia of the surrounding tissue. Subsequent upregulation of HIF- $1 \alpha$ augments a milieu of pro-survival factors, including VEGF. VEGF overexpression yields aberrant neovascularization, which is highly permeable, resulting in perilesional edema and, ultimately, radiation necrosis [81]. Importantly, astrocytic glioma lineages have pronounced VEGF-mediated pathologic sequelae [47]. Furthermore, hierarchical grading of glioma tumors is strongly associated with VEGF expression predominating in GBM tumors [11].

Preclinical evidence describes a significant reduction in tumor volume and vascularization following RT with BVZ, which likely explains the reduced rates of RN in novel HGG patients [5, 27, 28, 39, 40]. BVZ exhibits radio-sensitizing effects by selectively targeting glial stem cells, which are otherwise minimally neutralized by radiation $[4,5]$. BVZ thereby limits aberrant revascularization, a key mechanism in restricting further tumor growth [4, 25, 39]. By reducing VEGF expression, BVZ helps establish a normoxic niche, enhancing the cytotoxicity of radiation therapy $[5,28]$. Thus, concomitant BVZ and irradiation have an advantageous 


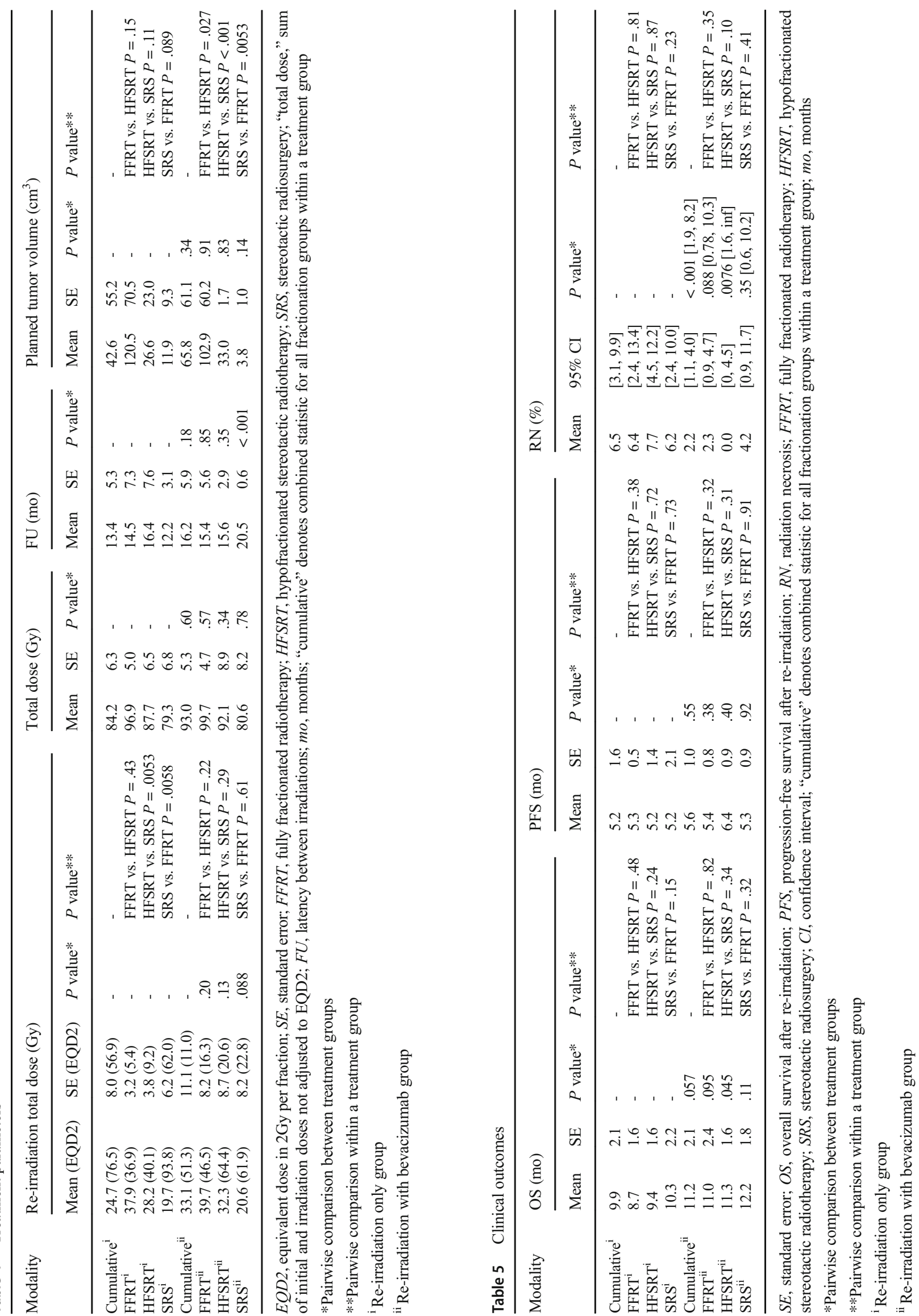


Table 6 Multivariate linear regression analysis for overall survival after re-irradiation

\begin{tabular}{llll}
\hline Explanatory variable & Beta value & $95 \%$ CI & $P$ value \\
\hline Age & 0.23 & {$[-0.11,0.57]$} & .16 \\
GBM & 0.43 & {$[-3.76,4.63]$} & .83 \\
HFSRT & -1.22 & {$[-4.64,2.20]$} & .46 \\
FFRT & 0.24 & {$[-4.77,5.25]$} & .92 \\
Bevacizumab & 2.51 & {$[0.11,4.92]$} & .041 \\
FU & 0.0066 & {$[-0.24,0.25]$} & .95 \\
PTV & -0.023 & {$[-0.066,0.018]$} & .24 \\
EQD2 RT & 0.41 & {$[-0.62,1.43]$} & .41 \\
EQD2 reRT & 0.0186 & {$[-0.039,0.076]$} & .50 \\
\hline
\end{tabular}

GBM, glioblastoma; HFSRT, hypofractionated stereotactic radiotherapy; $F F R T$, fully fractionated radiotherapy; $F U$, latency between irradiations; $P T V$, planned tumor volume; $R T$, initial radiotherapy; $r e R T$, re-irradiation therapy; $E Q D 2$, equivalent dose in $2 \mathrm{~Gy}$ per fraction; $C I$, confidence interval

synergistic therapeutic effect compared to BVZ administration alone [28, 39].

Clinically, there have been conflicting reports on the therapeutic efficacy of adjuvant BVZ in recurrent HGG patients. Our systematic review found that reRT + BVZ improves both $\mathrm{RN}$ and $\mathrm{OS}$ when compared to reRT alone. In a recent study, Fleischmann et al. [23] demonstrated that BVZ was significantly associated with decreased radiation necrosis and edema rates. Consistent with our data, Fleischmann et al. reported significant reductions in RN using BVZ in both univariate and multivariate analyses. Additionally, several other publications corroborate a moderate side effect profile for BVZ, with low or acceptable reported toxicity rates. Among studies included in our review, the reRT-only and reRT + BVZ

Table 7 Multivariate linear regression analysis for progression-free survival after re-irradiation

\begin{tabular}{llll}
\hline Explanatory variable & Beta value & $95 \%$ CI & $P$ value \\
\hline Age & 0.11 & {$[-0.089,0.30]$} & .23 \\
GBM & 1.36 & {$[-1.21,3.94]$} & .24 \\
HFSRT & 1.17 & {$[-1.41,3.75]$} & .31 \\
FFRT & 0.71 & {$[-3.23,4.67]$} & .67 \\
Bevacizumab & 1.40 & {$[-0.36,3.18]$} & .099 \\
FU & 0.020 & {$[-0.18,0.21]$} & .81 \\
PTV & -0.00012 & {$[-0.036,0.036]$} & .99 \\
EQD2 RT & 0.080 & {$[-0.59,0.75]$} & .78 \\
EQD2 reRT & 0.015 & {$[-0.032,0.061]$} & .47 \\
\hline
\end{tabular}

GBM, glioblastoma; HFSRT, hypofractionated stereotactic radiotherapy; $F F R T$, fully fractionated radiotherapy; $F U$, latency between irradiations; $P T V$, planned tumor volume; $R T$, initial radiotherapy; $r e R T$, re-irradiation therapy; $E Q D 2$, equivalent dose in $2 \mathrm{~Gy}$ per fraction; $C I$, confidence interval treatment group had comparable toxicity rates of $21 \%$ and $25 \%$, respectively.

Superior survival and functional outcomes are more commonly reported for women than men with HGG [73]. Stratified by re-radiation modality, gender distributions were comparable for FFRT (35\% female) and HFSRT (28\% female), reflecting known demographics for HGG patient populations in general [73]. However, the gender demographics among patients receiving SRS differed significantly between reRT and reRT $+\mathrm{BVZ}$ treatment groups $(P=.002$, Table 3$)$. Despite a higher proportion of male patients, the median KPS score among our pooled reRT + BVZ SRS cohort was significantly higher than the reRT SRS cohort. Such inconsistency indicates a predilection for better-performing males in the reRT + BVZ group, limiting the interpretation of outcome differences for our pooled SRS cohorts.

Our reRT + BVZ groups had a higher proportion of grade III gliomas. While grade III gliomas are associated with favorable clinical outcomes compared to GBM [50], it is unclear whether BVZ portends a particular benefit for patients with grade III gliomas relative to patients with GBM. However, interpretation of BVZ's role in outcomes for patients with grade III gliomas is limited, considering the updated WHO glioma classification [41]. In fact, IDH wild-type anaplastic astrocytomas have been shown to have similar clinical and molecular behavior as IDH wild-type GBM $[9,60]$. Still, IDH wild-type AA constitutes the minority of grade III AA [60], and tumor grade was not associated with OS, PFS, or RN rate in our comparative univariate analyses. Similarly, tumor grade was not significantly associated with survival outcomes in our multivariate analysis. Most important, we found a significant beneficial effect of BVZ treatment on overall survival after adjusting for tumor grade in a multivariate linear regression analysis.

Balancing planned radiation treatment volumes with appropriate radiation doses is paramount to achieving safe and effective treatment. Higher doses destroy tumors more efficiently, albeit with a higher risk of side effects, particularly radiation necrosis [38]. In our pooled cohorts, there were no differences in total reRT dose between reRT- and reRT + BVZ-treated patients, whether they received SRS, HFSRT, or FFRT. Improved outcomes may be attainable with more aggressive radiation dosages in the setting of BVZ, given BVZ's radioprotective effects [23, 27, 40]. To this point, both Clarke et al. [14] and Schernberg et al. [61] discuss the possibility of using BVZ to permit higher radiation doses with SRS and FFRT without undue radiation toxicity. Clarke et al. demonstrated an acceptable toxicity profile of a more aggressive SRS regimen (33 Gy in 3 fractions, EQD2 = 107 Gy) compared to a lower dose regimen $(30 \mathrm{~Gy}$ in 5 fractions, EQD2 = 60 Gy). Similarly, Schernberg et al. reported improved survival associated with a more aggressive FFRT regimen for reRT (EQD2 > 50 Gy) compared to less aggressive dosing. 
Finally, Yasuda et al. [79] reported no radiation necrosis in patients with a more aggressive HFSRT radiation regimen of 42 Gy in 7 fractions (EQD2 $=84$ Gy) in combination with BVZ. Notably, these authors described acceptable toxicity profiles despite exceeding cumulative doses over $100 \mathrm{~Gy}$, a range traditionally associated with increased risk of radiation necrosis in the absence of BVZ $[43,67]$. While we did not find a significant effect of the total dose (i.e., EQD2) at reirradiation on patient outcomes, more rigorous studies are required to investigate the optimal volume range and radiation dose for each fractionation regimen supplemented with BVZ.

\section{Limitations}

The retrospective nature of the majority of available studies limited our systematic review [70]. Additionally, the paucity of literature for reRT + BVZ treatment involving HFSRT and SRS limited our assessment of BVZ and reRT modality on clinical outcomes. Several papers reported combined data for HGG tumors and did not delineate between grade III and IV gliomas. Moreover, the heterogeneity in data reporting and treatment protocols prevented us from disaggregating important demographic, treatment, and clinical outcome variables in some cases. Partial or inconsistent reporting of BVZ-induced toxicities also prevented us from performing any rigorous synthesis of adverse outcomes from BVZ in this analysis.

Overall, study heterogeneity and risk of bias were thus major limitations of our approach and are inherent in any systematic review or meta-analysis. We attempted to address both these issues using robust meta-analytic modeling techniques (Supplementary Tables 1-5). Our RoB analyses demonstrated that obvious sources of study bias did not systematically skew reported study outcomes. However, we found that study heterogeneity was present within multiple treatment groups for both RN and OS outcomes. Comparing our simple pooling of median OS and $\mathrm{RN}$ rates using weighted means (Table 5) with the more rigorous meta-analytic quantification of pooled estimates incorporating random study effects (Supplementary Tables 1-2) in general showed good agreement in the pooled survival and $\mathrm{RN}$ estimates. The only major exception was OS in the FFRT group (8.7 months using weighted means vs. 14.3 months using a random-effects model). This discrepancy is explained simply by the fact that many studies did not report sufficient data to be amenable to the meta-analysis of median survival times (see Methods); thus, only a subset of reviewed studies contributed to the data reported in Supplementary Table 2. Still, our findings provide the most comprehensive synthesis for recurrent glioma outcomes after reRT - given the limitations in data reported in the existing literature - and suggest a possible benefit of BVZ for both OS and RN. However, improved estimation of clinical outcomes will require improved data reporting from clinical studies, which would ideally involve comprehensive datasets reported to shared electronic databases. Continued innovation in meta-analytic techniques may also push the limits of what can be achieved with the existing literature. For example, direct graphical estimation of individual data points from published survival curves might be used to glean more granular data from individual studies even when not reported directly by authors in the text. While not attempted here, such an approach may become more feasible in future work utilizing novel analysis software being pioneered for this purpose $[52,58]$.

Regarding radiation necrosis, accurate evaluation of antiVEGF therapy response is also limited by imaging techniques, as conventional MRI does not reliably differentiate between pseudoprogression, tumor progression, and RN [78]. However, dynamic contrast-enhanced (DCE) perfusion MRI and diffusion-weighted imaging (DWI) are better able to differentiate between post-treatment radiation effect and actual tumor progression [21, 32, 49, 55]. Decreased tumor permeability and perfusion detected on DCE-MRI can reliably correlate with improved clinical outcomes after BVZ treatment $[8,29]$. Such imaging results suggest that BVZ's effects are consistent with the proposed vascular modulating theory. Recent reviews have further justified the more consistent use of perfusion MRI technology for evaluating HGG patients $[49,55,61]$. The lack of uniform objective characterization of tumor progression limits reliable determination of PFS and the potential benefits of novel therapeutic agents in slowing disease progression [12]. These limitations may have contributed to the lack of a significant benefit of BVZ on PFS in our multivariate analysis. As DCE and DWI technology improves, guidelines should be updated to standardize radiologic determination of tumor progression and RN.

Finally, most publications included in this study predated the revised 2016 WHO classification of CNS tumors; thus, IDH classification was not consistently reported. While this limits our interpretation of outcomes according to current standards, our study's comparative nature does elucidate general correlations according to a classically utilized grading schema. Future studies should consistently report and compare the effect of BVZ on recurrent gliomas characterized by the updated 2016 WHO schema for gliomas.

\section{Conclusion}

To our knowledge, this study is the most extensive and targeted systematic review evaluating the impact of bevacizumab on clinical outcomes in the setting of recurrent HGG treated with reRT. Our results suggest that reRT + BVZ may be associated with improved OS and lower $\mathrm{RN}$ rates than reRT alone. Upon multivariate analysis, treatment with BVZ was the only clinical variable significantly associated with improved OS. reRT + BVZ treatment had the most 
pronounced benefits in patients receiving HFSRT. Our findings suggest a potential benefit for reRT + BVZ, yet are limited by inconsistent data reporting and heterogeneity in study methodologies, the latter of which was also reflected in the heterogeneity of OS and RN outcomes reported across studies describing the same patient subgroups. Our findings support further randomized prospective studies to robustly assess the potential benefit of BVZ in patients with glioma and highlight the need for improved outcome reporting of published studies in this area. Future studies should clarify optimal reRT protocols and BVZ regimens (i.e., timing, dosing, and treatment duration). Finally, further work is needed to more accurately diagnose radiologic progression and $\mathrm{RN}$ in patients with recurrent glioma undergoing reRT.

Supplementary Information The online version contains supplementary material available at https://doi.org/10.1007/s00701-021-04794-3.

Funding Aditya Kondajji, Thien Nguyen, and John P. Sheppard are recipients of the David Geffen Medical Scholarship. Kunal Patel is supported by an NIH R25 grant. Dr. Isaac Yang reports being supported by the UCLA Visionary Ball Fund Grant, Eli and Edythe Broad Center of Regenerative Medicine and Stem Cell Research UCLA Scholars in Translational Medicine Program Award, Jason Dessel Memorial Seed Grant, UCLA Honberger Endowment Brain Tumor Research Seed Grant, and Stop Cancer (US) Development Award. The sponsors had no role in the design or conduct of this research.

\section{Declaration}

Conflict of interest The authors declare no conflict of interest.

Open Access This article is licensed under a Creative Commons Attribution 4.0 International License, which permits use, sharing, adaptation, distribution and reproduction in any medium or format, as long as you give appropriate credit to the original author(s) and the source, provide a link to the Creative Commons licence, and indicate if changes were made. The images or other third party material in this article are included in the article's Creative Commons licence, unless indicated otherwise in a credit line to the material. If material is not included in the article's Creative Commons licence and your intended use is not permitted by statutory regulation or exceeds the permitted use, you will need to obtain permission directly from the copyright holder. To view a copy of this licence, visit http://creativecommons.org/licenses/by/4.0/.

\section{References}

1. Amelio D, Amichetti M (2012) Radiation therapy for the treatment of recurrent glioblastoma: an overview. Cancers 4:257. https://doi. org/10.3390/cancers4010257

2. Auffinger B, Spencer D, Pytel P, Ahmed AU, Lesniak MS (2015) The role of glioma stem cells in chemotherapy resistance and glioblastoma multiforme recurrence. Expert Rev Neurother 15:741752. https://doi.org/10.1586/14737175.2015.1051968

3. Back M, Gzell CE, Kastelan M, Guo L, Wheeler HR (2015) Large volume re-irradiation with bevacizumab is a feasible salvage option for patients with refractory high-grade glioma. In: Neurooncol
Pract, vol 2. vol 1. pp 48-53. doi:https://doi.org/10.1093/nop/ npu031

4. Bao S, Wu Q, McLendon RE, Hao Y, Shi Q, Hjelmeland AB, Dewhirst MW, Bigner DD, Rich JN (2006) Glioma stem cells promote radioresistance by preferential activation of the DNA damage response. Nature 444:756-760. https://doi.org/10.1038/ nature 05236

5. Bao S, Wu Q, Sathornsumetee S, Hao Y, Li Z, Hjelmeland AB, Shi Q, McLendon RE, Bigner DD, Rich JN (2006) Stem cell-like glioma cells promote tumor angiogenesis through vascular endothelial growth factor. Cancer Res 66:7843-7848. https://doi.org/10.1158/ 0008-5472.can-06-1010

6. Bir SC, Connor DE Jr, Ambekar S, Wilden JA, Nanda A (2015) Factors predictive of improved overall survival following stereotactic radiosurgery for recurrent glioblastoma. Neurosurg Rev 38:705713. https://doi.org/10.1007/s10143-015-0632-4

7. Biswas T, Okunieff P, Schell MC, Smudzin T, Pilcher WH, Bakos RS, Vates GE, Walter KA, Wensel A, Korones DN, Milano MT (2009) Stereotactic radiosurgery for glioblastoma: retrospective analysis. Radiat Oncol 4:11. https://doi.org/10.1186/1748-717X4-11

8. Cabrera AR, Cuneo KC, Desjardins A, Sampson JH, McSherry F, Herndon JE 2nd, Peters KB, Allen K, Hoang JK, Chang Z, Craciunescu O, Vredenburgh JJ, Friedman HS, Kirkpatrick JP (2013) Concurrent stereotactic radiosurgery and bevacizumab in recurrent malignant gliomas: a prospective trial. Int $\mathrm{J}$ Radiat Oncol Biol Phys 86:873-879. https://doi.org/10.1016/j.ijrobp. 2013.04.029

9. Cancer Genome Atlas Research N, Brat DJ, Verhaak RG, Aldape KD, Yung WK, Salama SR, Cooper LA, Rheinbay E, Miller CR, Vitucci M, Morozova O, Robertson AG, Noushmehr H, Laird PW, Cherniack AD, Akbani R, Huse JT, Ciriello G, Poisson LM, Barnholtz-Sloan JS, Berger MS, Brennan C, Colen RR, Colman $\mathrm{H}$, Flanders AE, Giannini C, Grifford M, Iavarone A, Jain R, Joseph I, Kim J, Kasaian K, Mikkelsen T, Murray BA, O'Neill BP, Pachter L, Parsons DW, Sougnez C, Sulman EP, Vandenberg SR, Van Meir EG, von Deimling A, Zhang H, Crain D, Lau K, Mallery D, Morris S, Paulauskis J, Penny R, Shelton T, Sherman M, Yena P, Black A, Bowen J, Dicostanzo K, Gastier-Foster J, Leraas KM, Lichtenberg TM, Pierson CR, Ramirez NC, Taylor C, Weaver S, Wise L, Zmuda E, Davidsen T, Demchok JA, Eley G, Ferguson ML, Hutter CM, Mills Shaw KR, Ozenberger BA, Sheth M, Sofia HJ, Tarnuzzer R, Wang Z, Yang L, Zenklusen JC, Ayala B, Baboud J, Chudamani S, Jensen MA, Liu J, Pihl T, Raman R, Wan Y, Wu Y, Ally A, Auman JT, Balasundaram M, Balu S, Baylin SB, Beroukhim R, Bootwalla MS, Bowlby R, Bristow CA, Brooks D, Butterfield Y, Carlsen R, Carter S, Chin L, Chu A, Chuah E, Cibulskis K, Clarke A, Coetzee SG, Dhalla N, Fennell T, Fisher S, Gabriel S, Getz G, Gibbs R, Guin R, Hadjipanayis A, Hayes DN, Hinoue T, Hoadley K, Holt RA, Hoyle AP, Jefferys SR, Jones S, Jones CD, Kucherlapati R, Lai PH, Lander E, Lee S, Lichtenstein L, Ma Y, Maglinte DT, Mahadeshwar HS, Marra MA, Mayo M, Meng S, Meyerson ML, Mieczkowski PA, Moore RA, Mose LE, Mungall AJ, Pantazi A, Parfenov M, Park PJ, Parker JS, Perou CM, Protopopov A, Ren X, Roach J, Sabedot TS, Schein J, Schumacher SE, Seidman JG, Seth S, Shen H, Simons JV, Sipahimalani P, Soloway MG, Song X, Sun H, Tabak B, Tam A, Tan D, Tang J, Thiessen N, Triche T Jr, Van Den Berg DJ, Veluvolu U, Waring S, Weisenberger DJ, Wilkerson MD, Wong T, Wu J, Xi L, Xu AW, Yang L, Zack TI, Zhang J, Aksoy BA, Arachchi H, Benz C, Bernard B, Carlin D, Cho J, DiCara D, Frazer S, Fuller GN, Gao J, Gehlenborg N, Haussler D, Heiman DI, Iype L, Jacobsen A, Ju Z, Katzman S, Kim H, Knijnenburg T, Kreisberg RB, Lawrence MS, Lee W, Leinonen K, Lin P, Ling S, Liu W, Liu Y, Liu Y, Lu Y, Mills G, Ng S, Noble MS, Paull E, Rao A, Reynolds S, Saksena G, Sanborn Z, 
Sander C, Schultz N, Senbabaoglu Y, Shen R, Shmulevich I, Sinha R, Stuart J, Sumer SO, Sun Y, Tasman N, Taylor BS, Voet D, Weinhold N, Weinstein JN, Yang D, Yoshihara K, Zheng S, Zhang W, Zou L, Abel T, Sadeghi S, Cohen ML, Eschbacher J, Hattab EM, Raghunathan A, Schniederjan MJ, Aziz D, Barnett G, Barrett W, Bigner DD, Boice L, Brewer C, Calatozzolo C, Campos B, Carlotti CG Jr, Chan TA, Cuppini L, Curley E, Cuzzubbo S, Devine K, DiMeco F, Duell R, Elder JB, Fehrenbach A, Finocchiaro G, Friedman W, Fulop J, Gardner J, Hermes B, Herold-Mende C, Jungk C, Kendler A, Lehman NL, Lipp E, Liu O, Mandt R, McGraw M, McLendon R, McPherson C, Neder L, Nguyen P, Noss A, Nunziata R, Ostrom QT, Palmer C, Perin A, Pollo B, Potapov A, Potapova O, Rathmell WK, Rotin D, Scarpace L, Schilero C, Senecal K, Shimmel K, Shurkhay V, Sifri S, Singh R, Sloan AE, Smolenski K, Staugaitis SM, Steele R, Thorne L, Tirapelli DP, Unterberg A, Vallurupalli M, Wang Y, Warnick R, Williams F, Wolinsky Y, Bell S, Rosenberg M, Stewart C, Huang F, Grimsby JL, Radenbaugh AJ, Zhang J (2015) Comprehensive, integrative genomic analysis of diffuse lower-grade gliomas. N Engl J Med 372:2481-2498. https://doi.org/10.1056/ NEJMoa1402121

10. Chamberlain MC, Barba D, Kormanik P, Shea WM (1994) Stereotactic radiosurgery for recurrent gliomas. Cancer 74:1342 1347. https://doi.org/10.1002/1097-0142(19940815)74:4<1342:: aid-cncr2820740426>3.0.co;2-y

11. Chaudhry IH, O'Donovan DG, Brenchley PE, Reid H, Roberts IS (2001) Vascular endothelial growth factor expression correlates with tumour grade and vascularity in gliomas. Histopathology 39 : 409-415. https://doi.org/10.1046/j.1365-2559.2001.01230.x

12. Chukwueke UN, Wen PY (2019) Use of the Response Assessment in Neuro-Oncology (RANO) criteria in clinical trials and clinical practice. CNS Oncol 8. https://doi.org/10.2217/cns-2018-0007

13. Ciammella P, Podgornii A, Galeandro M, D'Abbiero N, Pisanello A, Botti A, Cagni E, Iori M, Iotti C (2013) Hypofractionated stereotactic radiation therapy for recurrent glioblastoma: single institutional experience. Radiat Oncol 8:222. https://doi.org/10.1186/ 1748-717X-8-222

14. Clarke J, Neil E, Terziev R, Gutin P, Barani I, Kaley T, Lassman AB, Chan TA, Yamada J, DeAngelis L, Ballangrud A, Young R, Panageas KS, Beal K, Omuro A (2017) Multicenter, phase 1, dose escalation study of hypofractionated stereotactic radiation therapy with bevacizumab for recurrent glioblastoma and anaplastic astrocytoma. Int J Radiat Oncol Biol Phys 99:797-804. https://doi.org/ 10.1016/j.ijrobp.2017.06.2466

15. Combs SE, Gutwein S, Thilmann C, Huber P, Debus J, SchulzErtner D (2005) Stereotactically guided fractionated re-irradiation in recurrent glioblastoma multiforme. J Neurooncol 74:167-171. https://doi.org/10.1007/s11060-004-2463-y

16. Combs SE, Widmer V, Thilmann C, Hof H, Debus J, Schulz-Ertner D (2005) Stereotactic radiosurgery (SRS): treatment option for recurrent glioblastoma multiforme (GBM). Cancer 104:2168-2173. https://doi.org/10.1002/cncr.21429

17. Combs SE, Bischof M, Welzel T, Hof H, Oertel S, Debus J, SchulzErtner D (2008) Radiochemotherapy with temozolomide as reirradiation using high precision fractionated stereotactic radiotherapy (FSRT) in patients with recurrent gliomas. Journal of NeuroOncology 89:205-210. https://doi.org/10.1007/s11060-008-96074

18. Cuneo KC, Vredenburgh JJ, Sampson JH, Reardon DA, Desjardins A, Peters KB, Friedman HS, Willett CG, Kirkpatrick JP (2012) Safety and efficacy of stereotactic radiosurgery and adjuvant bevacizumab in patients with recurrent malignant gliomas. Int $\mathrm{J}$ Radiat Oncol Biol Phys 82:2018-2024. https://doi.org/10.1016/j. ijrobp.2010.12.074
19. de Groot JF (2015) High-grade gliomas. Continuum (Minneap Minn) 21:332-344. https://doi.org/10.1212/01.CON.0000464173. 58262.d9

20. Dincoglan F, Beyzadeoglu M, Sager O, Demiral S, Gamsiz H, Uysal B, Ebruli C, Akin M, Oysul K, Sirin S, Dirican B (2015) Management of patients with recurrent glioblastoma using hypofractionated stereotactic radiotherapy. Tumori 101:179-184. https://doi.org/10.5301/tj.5000236

21. Durand-Muñoz C, Flores-Alvarez E, Moreno-Jimenez S, RoldanValadez E (2019) Pre-operative apparent diffusion coefficient values and tumour region volumes as prognostic biomarkers in glioblastoma: correlation and progression-free survival analyses. In: Insights Imaging, vol 10. doi:https://doi.org/10.1186/s13244019-0724-8

22. Elliott RE, Parker EC, Rush SC, Kalhorn SP, Moshel YA, Narayana A, Donahue B, Golfinos JG (2011) Efficacy of gamma knife radiosurgery for small-volume recurrent malignant gliomas after initial radical resection. World Neurosurg 76:128-140; discussion 161-122. https://doi.org/10.1016/j.wneu.2010.12.053

23. Fleischmann DF, Jenn J, Corradini S, Ruf V, Herms J, Forbrig R, Unterrainer M, Thon N, Kreth FW, Belka C, Niyazi M (2019) Bevacizumab reduces toxicity of reirradiation in recurrent highgrade glioma. Radiother Oncol 138:99-105. https://doi.org/10. 1016/j.radonc.2019.06.009

24. Fowler JF (2010) 21 years of biologically effective dose. In: Br J Radiol, vol 83. vol 991. pp 554-568. doi:https://doi.org/10.1259/ bjr $/ 31372149$

25. Garcia-Barros M, Paris F, Cordon-Cardo C, Lyden D, Rafii S, Haimovitz-Friedman A, Fuks Z, Kolesnick R (2003) Tumor response to radiotherapy regulated by endothelial cell apoptosis. Science 300:1155-1159. https://doi.org/10.1126/science.1082504

26. Gigliotti MJ, Hasan S, Karlovits SM, Ranjan T, Wegner RE (2018) Re-irradiation with stereotactic radiosurgery/radiotherapy for recurrent high-grade gliomas: improved survival in the modern era. Stereotact Funct Neurosurg 96:289-295. https://doi.org/10.1159/ 000493545

27. Gonzalez J, Kumar AJ, Conrad CA, Levin VA (2007) Effect of bevacizumab on radiation necrosis of the brain. Int J Radiat Oncol Biol Phys 67:323-326. https://doi.org/10.1016/j.ijrobp.2006.10. 010

28. Gorski DH, Beckett MA, Jaskowiak NT, Calvin DP, Mauceri HJ, Salloum RM, Seetharam S, Koons A, Hari DM, Kufe DW, Weichselbaum RR (1999) Blockage of the vascular endothelial growth factor stress response increases the antitumor effects of ionizing radiation. Cancer Res 59:3374-3378

29. Gutin PH, Iwamoto FM, Beal K, Mohile NA, Karimi S, Hou BL, Lymberis S, Yamada Y, Chang J, Abrey LE (2009) Safety and efficacy of bevacizumab with hypofractionated stereotactic irradiation for recurrent malignant gliomas. Int J Radiat Oncol Biol Phys 75:156-163. https://doi.org/10.1016/j.ijrobp.2008.10.043

30. Hall WA, Djalilian HR, Sperduto PW, Cho KH, Gerbi BJ, Gibbons JP, Rohr M, Clark HB (1995) Stereotactic radiosurgery for recurrent malignant gliomas. J Clin Oncol 13:1642-1648. https://doi. org/10.1200/JCO.1995.13.7.1642

31. Holt DE, Bernard ME, Quan K, Clump DA, Engh JA, Burton SA, Heron DE (2016) Salvage stereotactic radiosurgery for recurrent glioblastoma multiforme with prior radiation therapy. J Cancer Res Ther 12:1243-1248. https://doi.org/10.4103/0973-1482. 199537

32. Hu LS, Eschbacher JM, Heiserman JE, Dueck AC, Shapiro WR, Liu S, Karis JP, Smith KA, Coons SW, Nakaji P, Spetzler RF, Feuerstein BG, Debbins J, Baxter LC (2012) Reevaluating the imaging definition of tumor progression: perfusion MRI quantifies recurrent glioblastoma tumor fraction, pseudoprogression, and radiation necrosis to predict survival. Neuro Oncol 14:919-930. https://doi.org/10.1093/neuonc/nos112 
33. Hudes RS, Corn BW, Werner-Wasik M, Andrews D, Rosenstock J, Thoron L, Downes B, Curran WJ Jr (1999) A phase I dose escalation study of hypofractionated stereotactic radiotherapy as salvage therapy for persistent or recurrent malignant glioma. Int $\mathrm{J}$ Radiat Oncol Biol Phys 43:293-298. https://doi.org/10.1016/s03603016(98)00416-7

34. Hundsberger T, Brugge D, Putora PM, Weder P, Weber J, Plasswilm L (2013) Re-irradiation with and without bevacizumab as salvage therapy for recurrent or progressive high-grade gliomas. J Neurooncol 112:133-139. https://doi.org/10.1007/s11060-0131044-3

35. Kazmi F, Soon YY, Leong YH, Koh WY, Vellayappan B (2019) Re-irradiation for recurrent glioblastoma (GBM): a systematic review and meta-analysis. Journal of Neuro-Oncology 142:79-90. https://doi.org/10.1007/s11060-018-03064-0

36. Khalil T, Lemaire JJ, Dedieu V, Donnarieix D, Beatrice C, Lapeyre M, Kemeny JL, Pereira B, Thalami A, Chazal J, Verrelle P (2013) MRI tumor response and clinical outcomes after LINAC radiosurgery on 50 patients with recurrent malignant gliomas. J Radiosurg SBRT 2:291-305

37. Kong DS, Lee JI, Park K, Kim JH, Lim DH, Nam DH (2008) Efficacy of stereotactic radiosurgery as a salvage treatment for recurrent malignant gliomas. Cancer 112:2046-2051. https://doi.org/ 10.1002/cncr.23402

38. Lawrence YR, Li XA, el Naqa I, Hahn CA, Marks LB, Merchant TE, Dicker AP (2010) RADIATION DOSE-VOLUME EFFECTS IN THE BRAIN. Int J Radiat Oncol Biol Phys 76:S20-S27. https:// doi.org/10.1016/j.ijrobp.2009.02.091

39. Lee CG, Heijn M, di Tomaso E, Griffon-Etienne G, Ancukiewicz M, Koike C, Park KR, Ferrara N, Jain RK, Suit HD, Boucher Y (2000) Anti-vascular endothelial growth factor treatment augments tumor radiation response under normoxic or hypoxic conditions. Cancer Res 60:5565-5570

40. Levin VA, Bidaut L, Hou P, Kumar AJ, Wefel JS, Bekele BN, Grewal J, Prabhu S, Loghin M, Gilbert MR, Jackson EF (2011) Randomized double-blind placebo-controlled trial of bevacizumab therapy for radiation necrosis of the central nervous system. Int $\mathrm{J}$ Radiat Oncol Biol Phys 79:1487-1495. https://doi.org/10.1016/j. ijrobp.2009.12.061

41. Louis DN, Perry A, Reifenberger G, von Deimling A, FigarellaBranger D, Cavenee WK, Ohgaki H, Wiestler OD, Kleihues P, Ellison DW (2016) The 2016 World Health Organization Classification of Tumors of the Central Nervous System: a summary. Acta Neuropathol 131:803-820. https://doi.org/10.1007/ s00401-016-1545-1

42. Martinez-Carrillo M, Tovar-Martin I, Zurita-Herrera M, Del MoralAvila R, Guerrero-Tejada R, Saura-Rojas E, Osorio-Ceballos JL, Arrebola-Moreno JP, Exposito-Hernandez J (2014) Salvage radiosurgery for selected patients with recurrent malignant gliomas. Biomed Res Int 2014:657953. https://doi.org/10.1155/2014/ 657953

43. Mayer R, Sminia P (2008) Reirradiation tolerance of the human brain. Int J Radiat Oncol Biol Phys 70:1350-1360. https://doi.org/ 10.1016/j.ijrobp.2007.08.015

44. McGrath S, Zhao X, Qin ZZ, Steele R, Benedetti A (2019) Onesample aggregate data meta-analysis of medians. Stat Med 38:969984. https://doi.org/10.1002/sim.8013

45. McGrath S, Zhao X, Steele R, Benedetti A (2020) metamedian: Meta-Analysis of Medians. The Comprehensive R Archive Network:1-10

46. Minniti G, Agolli L, Falco T, Scaringi C, Lanzetta G, Caporello P, Osti MF, Esposito V, Enrici RM (2015) Hypofractionated stereotactic radiotherapy in combination with bevacizumab or fotemustine for patients with progressive malignant gliomas. J Neurooncol 122:559-566. https://doi.org/10.1007/s11060-015$1745-\mathrm{x}$
47. Miyatake SI, Nonoguchi N, Furuse M, Yoritsune E, Miyata T, Kawabata S, Kuroiwa T (2015) Pathophysiology, diagnosis, and treatment of radiation necrosis in the brain. In: Neurol Med Chir (Tokyo), vol 55. vol 1. pp 50-59. doi:https://doi.org/10.2176/nmc. ra.2014-0188

48. Niyazi M, Brada M, Chalmers AJ, Combs SE, Erridge SC, Fiorentino A, Grosu AL, Lagerwaard FJ, Minniti G, Mirimanoff R-O, Ricardi U, Short SC, Weber DC, Belka C (2016) ESTROACROP guideline "target delineation of glioblastomas". Radiotherapy and Oncology 118:35-42. https://doi.org/10.1016/j. radonc.2015.12.003

49. Okuchi S, Rojas-Garcia A, Ulyte A, Lopez I, Ušinskienė J, Lewis M, Hassanein SM, Sanverdi E, Golay X, Thust S, PanovskaGriffiths J, Bisdas S (2019) Diagnostic accuracy of dynamic contrast-enhanced perfusion MRI in stratifying gliomas: a systematic review and meta-analysis. In: Cancer Med, vol 8. vol 12. pp 5564-5573. doi:https://doi.org/10.1002/cam4.2369

50. Ostrom QT, Cioffi G, Gittleman H, Patil N, Waite K, Kruchko C, Barnholtz-Sloan JS (2019) CBTRUS statistical report: primary brain and other central nervous system tumors diagnosed in the United States in 2012-2016. Neuro Oncol 21:v1-v100. https:// doi.org/10.1093/neuonc/noz150

51. Palmer JD, Bhamidipati D, Song A, Eldredge-Hindy HB, Siglin J, Dan TD, Champ CE, Zhang I, Bar-Ad V, Kim L, Glass J, Evans JJ, Andrews DW, Werner-Wasik M, Shi W (2018) Bevacizumab and re-irradiation for recurrent high grade gliomas: does sequence matter? Journal of Neuro-Oncology 140:623-628. https://doi.org/10. 1007/s11060-018-2989-Z

52. Pandey S (2020) MetaSurvival: Meta-Analysis of a single survival curve (version 0.1.0). https://CRAN.R-project.org/package= metaSurvival

53. Park DM, Rich JN (2009) Biology of glioma cancer stem cells. Mol Cells 28:7-12. https://doi.org/10.1007/s10059-009-0111-2

54. Pasek J, and with some assistance from Alex Tahk and some code modified from R.-core; Additional contributions by Gene Culter and Marcus Schwemmle (2020) Weights: Weighting and Weighted Statistics (version 1.0.1). https://CRAN.R-project.org/ package $=$ weights

55. Patel P, Baradaran H, Delgado D, Askin G, Christos P, John Tsiouris A, Gupta A (2017) MR perfusion-weighted imaging in the evaluation of high-grade gliomas after treatment: a systematic review and meta-analysis. Neuro Oncol 19:118-127. https://doi. org/10.1093/neuonc/now148

56. Pawlik TM, Keyomarsi K (2004) Role of cell cycle in mediating sensitivity to radiotherapy. International Journal of Radiation Oncology*Biology*Physics 59:928-942. https://doi.org/10.1016/ j.ijrobp.2004.03.005

57. Pinzi V, Orsi C, Marchetti M, Milanesi IM, Bianchi LC, DiMeco F, Cuccarini V, Farinotti M, Ferroli P, Finocchiaro G, Franzini A, Fumagalli M, Silvani A, Fariselli L (2015) Radiosurgery reirradiation for high-grade glioma recurrence: a retrospective analysis. Neurol Sci 36:1431-1440. https://doi.org/10.1007/s10072015-2172-7

58. Poisot $\mathrm{T}$ (2011) The digitize package: extracting numerical data from scatter-plots. The R Journal 3:25-26

59. R Core Team (2017) R: A language and environment for statistical computing. R Foundation for Statistical Computing, Vienna

60. Reuss DE, Kratz A, Sahm F, Capper D, Schrimpf D, Koelsche C, Hovestadt V, Bewerunge-Hudler M, Jones DTW, Schittenhelm J, Mittelbronn M, Rushing E, Simon M, Westphal M, Unterberg A, Platten M, Paulus W, Reifenberger G, Tonn JC, Aldape K, Pfister SM, Korshunov A, Weller M, Herold-Mende C, Wick W, Brandner S, von Deimling A (2015) Adult IDH wild type astrocytomas biologically and clinically resolve into other tumor entities. Acta Neuropathol 130:407-417 
61. Schernberg A, Dhermain F, Ammari S, Dumont SN, Domont J, Patrikidou A, Pallud J, Dezamis E, Deutsch E, Louvel G (2018) Reirradiation with concurrent bevacizumab for recurrent highgrade gliomas in adult patients. Cancer Radiother 22:9-16. https:// doi.org/10.1016/j.canrad.2017.06.013

62. Scholtyssek F, Zwiener I, Schlamann A, Seidel C, Meixensberger J, Bauer M, Hoffmann K-T, Combs SE, von Bueren AO, Kortmann R-D, Müller K (2013) Reirradiation in progressive high-grade gliomas: outcome, role of concurrent chemotherapy, prognostic factors and validation of a new prognostic score with an independent patient cohort. Radiat Oncol 8:161. https://doi.org/10.1186/1748717X-8-161

63. Schwarzer G (2007) meta: an R package for meta-analysis. R News: The Newsletter of the R Project 7:40-45

64. Shanker M, Chua B, Bettington C, Foote MC, Pinkham MB (2019) Re-irradiation for recurrent high-grade gliomas: a systematic review and analysis of treatment technique with respect to survival and risk of radionecrosis. Neurooncol Pract 6:144-155. https://doi.org/10. 1093/nop/npy019

65. Sharifzad F, Ghavami S, Verdi J, Mardpour S, Mollapour Sisakht M, Azizi Z, Taghikhani A, Łos MJ, Fakharian E, Ebrahimi M, Hamidieh AA (2019) Glioblastoma cancer stem cell biology: potential theranostic targets. Drug Resist Updat 42:35-45. https://doi. org/10.1016/j.drup.2018.03.003

66. Shaw E, Scott C, Souhami L, Dinapoli R, Kline R, Loeffler J, Farnan N (2000) Single dose radiosurgical treatment of recurrent previously irradiated primary brain tumors and brain metastases: final report of RTOG protocol 90-05. Int J Radiat Oncol Biol Phys 47:291-298. https://doi.org/10.1016/s0360-3016(99)00507-6

67. Shepherd SF, Laing RW, Cosgrove VP, Warrington AP, Hines F, Ashley SE, Brada M (1997) Hypofractionated stereotactic radiotherapy in the management of recurrent glioma. Int J Radiat Oncol Biol Phys 37:393-398. https://doi.org/10.1016/s03603016(96)00455-5

68. Shrieve DC, Alexander E, Wen PY, Fine HA, Kooy HM, Black PM, Loeffler JS (1995) Comparison of stereotactic radiosurgery and brachytherapy in the treatment of recurrent glioblastoma multiforme. Neurosurgery 36:275-284. https://doi.org/10.1227/ 00006123-199502000-00006

69. Skeie BS, Enger PO, Brogger J, Ganz JC, Thorsen F, Heggdal JI, Pedersen PH (2012) gamma knife surgery versus reoperation for recurrent glioblastoma multiforme. World Neurosurg 78:658-669. https://doi.org/10.1016/j.wneu.2012.03.024

70. Song JW, Chung KC (2010) Observational studies: cohort and case-control studies. Plast Reconstr Surg 126:2234-2242. https:// doi.org/10.1097/PRS.0b013e3181f44abc

71. Stupp R, Mason WP, van den Bent MJ, Weller M, Fisher B, Taphoorn MJB, Belanger K, Brandes AA, Marosi C, Bogdahn U, Curschmann J, Janzer RC, Ludwin SK, Gorlia T, Allgeier A, Lacombe D, Cairncross JG, Eisenhauer E, Mirimanoff RO (2005) Radiotherapy plus concomitant and adjuvant temozolomide for glioblastoma. New England Journal of Medicine 352:987-996. https://doi.org/10.1056/NEJMoa043330
72. Stupp R, Hegi ME, Mason WP, van den Bent MJ, Taphoorn MJB, Janzer RC, Ludwin SK, Allgeier A, Fisher B, Belanger K, Hau P, Brandes AA, Gijtenbeek J, Marosi C, Vecht CJ, Mokhtari K, Wesseling P, Villa S, Eisenhauer E, Gorlia T, Weller M, Lacombe D, Cairncross JG, Mirimanoff R-O, European Organisation for R, Treatment of Cancer Brain T, Radiation Oncology G, National Cancer Institute of Canada Clinical Trials G (2009) Effects of radiotherapy with concomitant and adjuvant temozolomide versus radiotherapy alone on survival in glioblastoma in a randomised phase III study: 5-year analysis of the EORTCNCIC trial. Lancet Oncol 10:459-466. https://doi.org/10.1016/ S1470-2045(09)70025-7

73. Tian M, Ma W, Chen Y, Yu Y, Zhu D, Shi J, Zhang Y (2018) Impact of gender on the survival of patients with glioblastoma. Biosci Rep 38. https://doi.org/10.1042/bsr20180752

74. Torok JA, Wegner RE, Mintz AH, Heron DE, Burton SA (2011) Re-irradiation with radiosurgery for recurrent glioblastoma multiforme. Technol Cancer Res Treat 10:253-258. https://doi. org/10.7785/tcrt.2012.500200

75. Viechtbauer W (2010) Conducting meta-analyses in R with the metafor package. J Stat Softw 36:1-48

76. Vordermark D, Kolbl O, Ruprecht K, Vince GH, Bratengeier K, Flentje M (2005) Hypofractionated stereotactic re-irradiation: treatment option in recurrent malignant glioma. BMC Cancer 5:55. https://doi.org/10.1186/1471-2407-5-55

77. Voynov G, Kaufman S, Hong T, Pinkerton A, Simon R, Dowsett R (2002) Treatment of recurrent malignant gliomas with stereotactic intensity modulated radiation therapy. Am J Clin Oncol 25:606611. https://doi.org/10.1097/00000421-200212000-00017

78. Wen PY, Chang SM, Van den Bent MJ, Vogelbaum MA, Macdonald DR, Lee EQ (2017) Response assessment in neurooncology clinical trials. In: J Clin Oncol, vol 35. vol 21. pp 24392449. doi:https://doi.org/10.1200/jco.2017.72.7511

79. Yasuda T, Muragaki Y, Nitta M, Miyamoto K, Oura Y, Henmi T, Noguchi S, Oda H, Saito T, Maruyama T, Atsuchi S, Miura N, Kawamata T (2018) Effectiveness of stereotactic radiotherapy and bevacizumab for recurrent high-grade gliomas: a potential therapy for isocitrate dehydrogenase wild-type recurrent high-grade gliomas. World Neurosurg 114:e1138-e1146. https://doi.org/10.1016/ j.wneu.2018.03.161

80. Zemlin A, Martens B, Wiese B, Merten R, Steinmann D (2018) Timing of re-irradiation in recurrent high-grade gliomas: a single institution study. J Neurooncol 138:571-579. https://doi.org/10. 1007/s11060-018-2824-6

81. Zhuang H, Shi S, Yuan Z, Chang JY (2019) Bevacizumab treatment for radiation brain necrosis: mechanism, efficacy and issues. Mol Cancer 18:21. https://doi.org/10.1186/s12943-019-0950-1

Publisher's note Springer Nature remains neutral with regard to jurisdictional claims in published maps and institutional affiliations. 